\title{
Wave dispersion and growth analysis of low-voltage grating Čerenkov amplifiers
}

\author{
J. Joe, J. Scharer, J. Booske, and B. McVeya) \\ Department of Electrical and Computer Engineering, University of Wisconsin, Madison, Wisconsin 53706
}

(Received 16 August 1993; accepted 30 September 1993)

\begin{abstract}
A theoretical and computational investigation of an electron sheet beam propagating over a grating structure in a rectangular waveguide is carried out. Regimes for low voltage Cerenkov amplifier operation are sought by examining the complex dispersion relation for hybrid waveguide modes in the slow wave structure, which includes sheet beam space-charge effects. A computer code is developed to examine the complex dispersion relation and growth rates for the wave modes. Mode competition is considered and methods to reduce it are presented. Briggs' criteria is utilized to examine absolute and convective wave growth for the forward wave, backward wave, and transition mode regimes of operation as a function of the beam, hybrid mode, and slow wave grating characteristics. An examination of the effects of beam spread on absolute and convective wave growth to determine regimes for amplifier operation is carried out. A modest Maxwellian beam spread is found to yield a regime of effective backward convective amplification, in addition to the purely convective growth characteristics for the forward wave mode case.
\end{abstract}

\section{INTRODUCTION}

Črenkov radiation is usually referred to as electromagnetic radiation generated by passing an electron beam through or near a dielectric surface, where the phase velocity of the wave is less than that in vacuum. ${ }^{1,2}$ In this paper, we consider an extension of this definition that includes any electromagnetic radiation generated from a beam-slow wave structure interaction, including a conducting grating.

The scattering of a monochromatic electromagnetic plane wave that impinges on an electron beam propagating close to a planar grating has been investigated for both diffuse and high electron beam density regimes. ${ }^{3-5}$ The scattering yields an amplified wave at the incident plane wave frequency and evanescent harmonics. A tunable coherent radiation source has been experimentally realized that utilizes a grating based oscillator referred to as an Orotron. ${ }^{6}$ The device is constructed by adding a mirror parallel to a metal grating. The interaction is between the electron beam and Bragg-scattered electromagnetic fast waves that bounce transverse to the beam between the grating and the mirror. A more conventional grating-based device, also called Orotron, employs a synchronous interaction between the beam and electromagnetic slow surface modes guided by the grating. ${ }^{7}$ Radiation ranging from 30 to $110 \mathrm{GHz}$ at power levels of $100 \mathrm{~W}$ to $2 \mathrm{~kW}$ with beam energies $>100 \mathrm{keV}$ has been observed with the latter device.

In this paper, a theoretical investigation of a gratingbased Čerenkov amplifier operating with a low-energy $(\leqslant 40 \mathrm{kV})$ sheet electron beam is presented. The sheet beam has a finite thickness and finite axial thermal velocity spread and is passed in close proximity to a conducting

\footnotetext{
a) On leave from Los Alamos National Laboratory, Los Alamos, New Mexico, 87545 .
}

grating waveguide structure. The aforementioned Orotron oscillator ${ }^{7}$ consists of a metal grating and top planar conductor aligned parallel to the metal grating. Due to the absence of side walls, the modes in the structure can be expanded in terms of pure transverse electric (TE) and transverse magnetic (TM) modes. In a rectangular grating waveguide structure, side walls are added that close the metal grating to the top plane conductor and guide the wave. In this structure, the modes can no longer be expanded in terms of pure TE and TM modes, but must be described in terms of hybrid modes. For our case the hybrid modes are described in terms of $\mathrm{TE}_{x m n},{ }^{8}$ where $x$ refers to the transverse dimension of the guide.

A sheet beam configuration is chosen for several reasons. It has a lower current density compared to that of a conventional pencil beam for the same amount of total beam current, since the sheet beam has a larger crosssectional area. Thus, for a low-energy beam and moderate power source, the sheet beam configuration provides an alternative to mitigate space-charge effects, which can degrade amplifier performance. The sheet beam configuration is naturally suited for the rectangular geometry and enhances the effective interaction between the hybrid normal modes in the rectangular grating guide and the electron beam, hence, providing larger gain for the amplifier. Recent studies have predicted that stable transport of a sheet beam in a periodic cusped magnetic (PCM) field is possible ${ }^{9}$ if the cusp period is small compared to the diocotron instability growth length.

In this paper, we will analyze the grating parameters for operation of a rectangular grating amplifier in the microwave/millimeter wave range with a low-energy sheet electron beam and determine the amplifier growth rate in the collective regime. In particular, close attention is focused on the physics of spatial (convective) versus temporal (absolute) wave growth. For an amplifier, convective wave growth is required. A rigorous wave growth analysis 
utilizing Briggs' criteria ${ }^{10,11}$ is carried out for the forward wave, the backward wave, and the transition mode regime to determine the physical conditions for convective growth. Landau damping is included in the analysis and is shown to reduce the wave growth, as expected. However, this Landau damping effect also appears to suppress absolute instabilities in the backward wave mode regime, allowing the device to operate as a backward wave amplifier.

The paper is outlined as follows. In Sec. II, the linear growth rate for a rectangular grating amplifier is calculated. In calculating the coupled dispersion relation, we also allow for a finite sheet beam thickness, a finite axial thermal velocity spread in the shcet bcam, and a finite beam-grating surface separation. We will examine mode competition between the fundamental and the first-higherorder hybrid mode and discuss methods of reducing the higher-order mode growth. The wave growth analysis is examined in Sec. III. To accomplish this analysis, a GRating AMPlifier (GRAMP) ${ }^{12}$ computer code has been developed. This code is capable of plotting either the uncoupled dispersion relation or evaluating linear growth rates by solving the complex roots for the complex coupled dispersion relation, and generating contour plots of $|D(\omega, k)|$ in the complex- $k$ plane for a given complex $\omega$. Briggs' criteria is utilized to analyze absolute and convective wave growth in the system. This analysis will be applied to the forward wave, backward wave, and the transition mode regimes for the Črenkov amplifier. In Sec. IV we provide a summary of the analysis.

\section{LINEAR DISPERSION AND GROWTH ANALYSIS FOR A ČERENKOV GRATING AMPLIFIER}

\section{A. Derivation}

We derive a complex dispersion relation that describes electron sheet beam propagation in a rectangular grating waveguide. The rectangular grating waveguide is created by inserting a uniform grating section on the bottom surface of a rectangular waveguide.

We consider the interaction between a slow-wave and an electron sheet beam propagating in a rectangular grating waveguide. The configuration for a rectangular grating waveguide structure with the sheet beam is shown in Fig. 1. The rectangular grating waveguide has a period $d$, groove width $s$, groove depth $p$, width $w$, and height $b$ from the grating floor to the top conductor. An electron sheet beam of thickness $t$ streams above the grating surface with a separation distance $\delta$. The sheet beam is assumed to be well confined in an infinite axial DC magnetic field. The sheet beam is injected at velocity $v_{0}$ and has a finite axial thermal velocity spread $v_{\text {th }}$. The axial velocity distribution is assumed to be a Maxwellian. The phase variation of the slow wave in the rectangular grating waveguide is assumed to be of the form $e^{-j\left(\omega t-k_{z} z\right)}$. One of the eigenmode solution sets in this structure is a transverse electric field relative to the $\mathbf{x}$ direction, $\mathrm{TE}_{x}$, which does not have an $E_{x}$ component and is a subset of the hybrid modes. ${ }^{8,13}$ Hybrid modes have both $\mathbf{E}$ and $\mathbf{H}$ along the direction of propagation (according to this paper notation, both $E_{z}$ and $H_{z}$

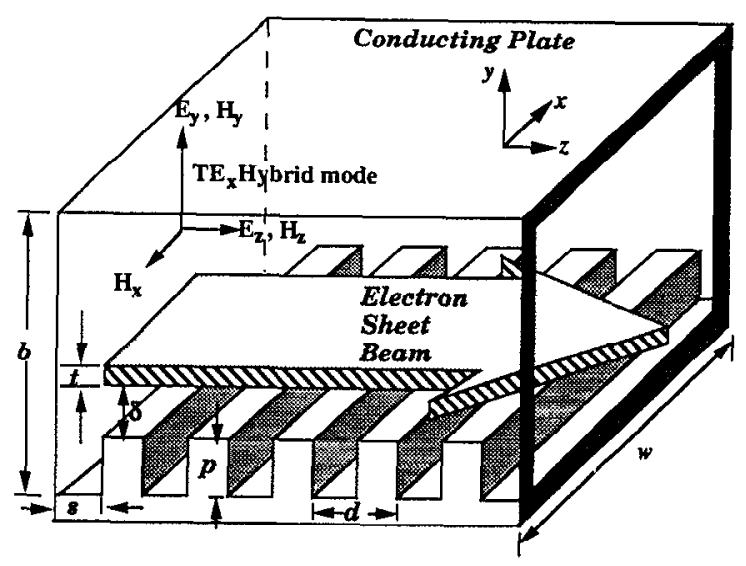

FIG. 1. An electron sheet beam passing over a rectangular grating waveguide.

exist). The components of the $\mathrm{TE}_{x}$ hybrid mode are shown in Fig. 1. For the $\mathrm{TE}_{x}$ mode, all vector components of the electromagnetic fields can be expressed in terms of $H_{x}$ by utilizing Maxwell's equations.

The field solution above the periodic grating structure is governed by Floquet's theorem. ${ }^{14}$ Hence, the magnetic field $H_{x}$ can be written as

$$
H_{x}(x, y, z)=\sum_{n=-\infty}^{n=\infty} H_{x n}(x, y) e^{-j\left(\omega t-k_{z n} z\right)}
$$

where $k_{z n}=k_{z}+2 \pi n / d$, and the subscript $n$ denotes the $n$th spatial harmonic. To satisfy the boundary condition $H_{x n}=0$ at $x=0$ and $x=w$ imposed by the conductor, we separate the variables in $H_{x n}$ and rewrite it as

$$
H_{x n}(x, y)=f_{n}(y) \sin \left(k_{x} x\right),
$$

where $f_{n}(y)$ is a function to be determined and $k_{x}=l \pi / w$, $l=1,2, \ldots$. The function $f_{n}(y)$ is obtained by solving a second-order differential equation derived from the Maxwell equations. It can be expressed in the vacuum region as follows:

$$
\frac{d^{2} f_{n}(y)}{d y^{2}}-v_{n}^{2} f_{n}(y)=0
$$

where $v_{n}^{2}=k_{z n}^{2}-v_{x}^{2}$. Here $v_{n}$ is the transverse wave number in the vacuum region and $v_{x}^{2}=k_{0}^{2}-k_{x}^{2}$ and $k_{0}=\omega / c$. In the beam region, we replace $v_{n}$ by $v_{r n}$, the transverse wave number in the beam region in Eq. (3), where $v_{m}^{2}=\left[\left(k_{0}^{2} \epsilon_{m n}-k_{x}^{2}\right) / v_{x}^{2}\right]\left(k_{z n}^{2}-v_{x}^{2}\right)$. The relative permittivity of the beam, $\epsilon_{r n}$, can be expressed as

$$
\epsilon_{r n}=1-\frac{\omega_{p e}^{2}}{k_{z n}^{2} v_{\mathrm{th}}^{2}} Z^{\prime}\left(\frac{k_{0}-k_{z n}\left(v_{0} / c\right)}{k_{z n}\left(v_{\mathrm{th}} / c\right)}\right),
$$

where $Z$ is the plasma dispersion function, $\omega_{p e}$ is the electron plasma frequency, and $v_{\text {th }}$ is the Maxwellian thermal spread.

Other $n$th harmonic field components can be obtained by solving the Maxwell equations with the following results: 


$$
\begin{aligned}
& E_{y n}=\frac{-\omega \mu_{0} k_{z}}{v_{x}^{2}} H_{x n}, \\
& E_{z n}=\frac{j \omega \mu_{0}}{k_{x}^{2}-k_{0}^{2} \epsilon_{r n}} \frac{\partial H_{x n}}{\partial y}, \\
& H_{y n}=\frac{-1}{k_{x}^{2}-k_{0}^{2} \epsilon_{r n}} \frac{\partial^{2} H_{x n}}{\partial x \partial y}, \\
& H_{z n}=\frac{j k_{z}}{v_{x}^{2}} \frac{\partial H_{x n}}{\partial x} .
\end{aligned}
$$

The rectangular grating is vertically divided into four layers or regions. Region I is defined by $0 \leqslant y \leqslant p, 0 \leqslant z \leqslant s$, region II by $p \leqslant y \leqslant p+\delta$, region III by $p+\delta \leqslant y \leqslant p+\delta+t$, and $p+\delta+t \leqslant y \leqslant b$ corresponds to region IV. Solving Eq. (3), then substituting $f_{n}(y)$ into Eqs. (2) and (1) and utilizing Eq. (6), we obtain the expressions for $H_{x n}$ and $E_{z n}$ in regions II, III, and IV, respectively, as

$$
\begin{aligned}
H_{x n}^{\mathrm{II}}= & {\left[B_{n} \cosh \left(v_{n} y\right)+C_{n} \sinh \left(v_{n} y\right)\right] \sin \left(k_{x} x\right), } \\
E_{z n}^{\mathrm{II}}= & \frac{-j \omega \mu_{0} v_{n}}{v_{x}^{2}}\left[B_{n} \sinh \left(v_{n} y\right)\right. \\
& \left.+C_{n} \cosh \left(v_{n} y\right)\right] \sin \left(k_{x} x\right), \\
H_{x n}^{\mathrm{III}}= & {\left[D_{n} \cosh \left(v_{r n} y\right)+E_{n} \sinh \left(v_{r n} y\right)\right] \sin \left(k_{x} x\right), } \\
E_{z n}^{\mathrm{III}}= & \frac{-j \omega \mu_{0} v_{r n}}{v_{r x n}^{2}}\left[D_{n} \sinh \left(v_{r n} y\right)\right. \\
& \left.+E_{n} \cosh \left(v_{r n} y\right)\right] \sin \left(k_{x} x\right), \\
H_{x n}^{\mathrm{IV}}= & F_{n} \cosh \left[v_{n}(b-y)\right] \sin \left(k_{x} x\right), \\
E_{z n}^{\mathrm{IV}}= & \frac{j \omega \mu_{0} v_{n}}{v_{x}^{2}} F_{n} \sinh \left[v_{n}(b-y)\right] \sin \left(k_{x} x\right),
\end{aligned}
$$

where $v_{r x n}^{2}=k_{0}^{2} \epsilon_{r n}-k_{x}^{2}$. Note that Eq. (11) satisfies the boundary conditions imposed by the top conductor, which requires that $E_{z n}^{\mathrm{IV}}=0$ at $y=b$. In region $\mathrm{I}$, the exact field solution is expanded in a Fourier series of the following form:

$$
H_{x}^{\mathrm{I}}=\sum_{m=0}^{m=\infty} A_{n} \cosh \left(v_{m} y\right) \cos \left(\frac{m \pi z}{s}\right) \sin \left(k_{x} x\right) .
$$

Note that $v_{m}^{2}=v_{x}^{2}-(m \pi / s)^{2}$ in region I. If we neglect higher-order modes in Eq. (12) (i.e., $m \geqslant 1$ ), we find by numerical computation that the dispersion relation roots are still in good agreement with those that include the higher-order modes. ${ }^{15}$ Therefore, we write the field expressions in region I solely in terms of the lowest-order mode, which can be written as follows:

$$
\begin{aligned}
& H_{x}^{\mathrm{I}}=A_{0} \sin \left(k_{x} x\right) \cos \left(v_{x} y\right), \\
& E_{z}^{\mathrm{I}}=\frac{j \omega \mu_{0}}{v_{x}} A_{0} \sin \left(k_{x} x\right) \sin \left(v_{x} y\right) .
\end{aligned}
$$

The field coefficients $C_{n}, D_{n}, E_{n}$, and $F_{n}$ are solved in terms of $B_{n}$ by applying the following tangential boundary conditions at the boundaries:
(1)
$H_{x n}^{\mathrm{IV}}=H_{x n}^{\mathrm{III}} \quad$ and $\quad E_{z n}^{\mathrm{IV}}=E_{z n}^{\mathrm{III}}, \quad$ at $y=p+\delta+t$,
(2) $H_{x n}^{\mathrm{III}}=H_{x n}^{\mathrm{II}}$ and $E_{z n}^{\mathrm{III}}=E_{z n}^{\mathrm{II}}$, at $y=p+\delta$.

We then rewrite the field solutions in region II in terms of $B_{n}$ as

$$
\begin{aligned}
H_{x}^{\mathrm{IL}}= & \sin \left(k_{x} x\right) \sum_{n=-\infty}^{n=\infty} B_{n}\left[\cosh \left(v_{n} y\right)\right. \\
& \left.+Y_{n} \sinh \left(v_{n} y\right)\right] e^{j k_{z n^{2}}} \\
E_{z}^{\mathrm{II}}= & \sin \left(k_{x} x\right) \sum_{n=-\infty}^{n=\infty} \frac{-j \omega \mu_{0} v_{n}}{v_{x}^{2}} B_{n}\left[\sinh \left(v_{n} y\right)\right. \\
& \left.+Y_{n} \cosh \left(v_{n} y\right)\right] e^{j k_{z n^{2}}}
\end{aligned}
$$

where

$$
\begin{aligned}
& Y_{n}=-\frac{v_{x}^{2} v_{r n} \Psi_{n}-v_{r x n}^{2} v_{n} \tanh \left[v_{n}(p+\delta)\right]}{v_{x}^{2} v_{r n} \Psi_{n} \tanh \left[v_{n}(p+\delta)\right]-v_{r x n}^{2} v_{n}}, \\
& \Psi_{n}=\frac{\tanh \left[v_{r n}(p+\delta)\right]-\Theta_{n}}{1-\Theta_{n} \tanh \left[v_{r n}(p+\delta)\right]}, \\
& \Theta_{n}=\frac{v_{r x n}^{2} v_{n} \tanh \left[v_{n}(b-\xi)\right]+v_{x}^{2} v_{r n} \tanh \left(v_{m} \xi\right)}{v_{r x n}^{2} v_{n} \tanh \left[v_{n}(b-\xi)\right] \tanh \left(v_{r n} \xi\right)+v_{x}^{2} v_{r n}}, \\
& \xi=p+\delta+t .
\end{aligned}
$$

The complex dispersion relation is obtained by imposing the boundary condition at the interface between regions I and II, which requires the continuity of $H_{x}$ and $E_{z}$ at $y=p, 0 \leqslant z \leqslant s$. At the top of the grating teeth, that is, at $y=p, s \leqslant z \leqslant d$, we require $E_{z}^{\mathrm{II}}=0$. By satisfying these boundary conditions, we obtain the complex dispersion relation as

$$
\begin{aligned}
D(\omega, k)= & 1-v_{x} d \tan \left(v_{x} p\right) \frac{s}{d} \\
& \times \sum_{n=-\infty}^{\infty} \frac{\sin c^{2}\left(k_{z n} s / 2\right)}{v_{n} d} \frac{\mathscr{N}_{n}(\omega, k)}{\mathscr{D}_{n}(\omega, k)},
\end{aligned}
$$

where

$$
\begin{aligned}
\mathscr{N}_{n}(\omega, k)= & 2\left(1+e^{-2 v_{r n} t}\right)\left(1+e^{-2 v_{n}(b-p-t)}\right)+\frac{v_{x}}{v_{r x n}} \\
& \times\left(1-e^{-2 v_{r n} t}\right)\left(\left(1+e^{-2 v_{n}(b-\xi)}\right)\left(1-e^{-2 v_{n} \delta}\right)\right. \\
& \left.+\frac{v_{r x n}^{2}}{v_{x}^{2}}\left(1-e^{-2 v_{n}(b-\xi)}\right)\left(1+e^{-2 v_{n} \delta}\right)\right) \\
\mathscr{D}_{n}(\omega, k)= & 2\left(1+e^{-2 v_{r n} t}\right)\left(1-e^{-2 v_{n}(b-p-t)}\right)+\frac{v_{x}}{v_{r x n}} \\
& \times\left(1-e^{-2 v_{r n} t}\right)\left(\left(1+e^{-2 v_{n}(b-\xi)}\right)\left(1+e^{-2 v_{n} \delta}\right)\right. \\
& \left.+\frac{v_{r x n}^{2}}{v_{x}^{2}}\left(1-e^{-2 v_{n}(b-\xi)}\right)\left(1-e^{-2 v_{n} \delta}\right)\right) .
\end{aligned}
$$

Joe et al. 


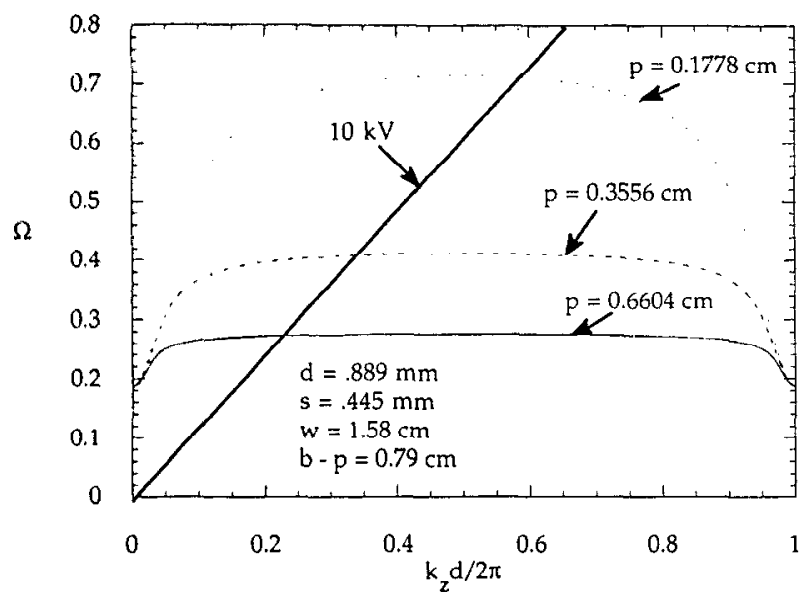

FIG. 2. Dispersion curves for the $\mathrm{TE}_{x 10}$ hybrid mode for various groove depths.

The vacuum grating structure dispersion relation can be obtained by reducing the beam thickness, $t$, to zero, which yields

$$
\begin{aligned}
D(\omega, k)= & 1-v_{x} d \tan \left(v_{x x} p\right) \frac{s}{d} \\
& \times \sum_{n=-\infty}^{\infty} \frac{\sin c^{2}\left(k_{z n} s / 2\right)}{v_{n} d \tanh \left[v_{n}(b-p)\right]} .
\end{aligned}
$$

Utilizing Eq. (18), the dispersion relation for various groove depths, $p$, and grating periods, $d$, for the fundamental $T E_{x 10}$ hybrid mode are displayed in Figs. 2 and 3, respectively. In the figures, $\Omega$ is normalized frequency and is equal to $k_{0} d$. We consider a $10 \mathrm{kV}$ electron beam energy for operation in the forward wave mode regime $\left(k_{z} d<0.5\right)$, which supports only convective (spatial) growth. Figure 2 shows that an increase in the groove depth, for constant period $d$, flattens and lowers the dispersion curve, similar to results obtained with the $\mathrm{TM}_{z}$ modes of the open parallel plate structure in Refs. 7 and 16. The intersection between the $10 \mathrm{kV}$ beamline and the dispersion curve is shifted to a

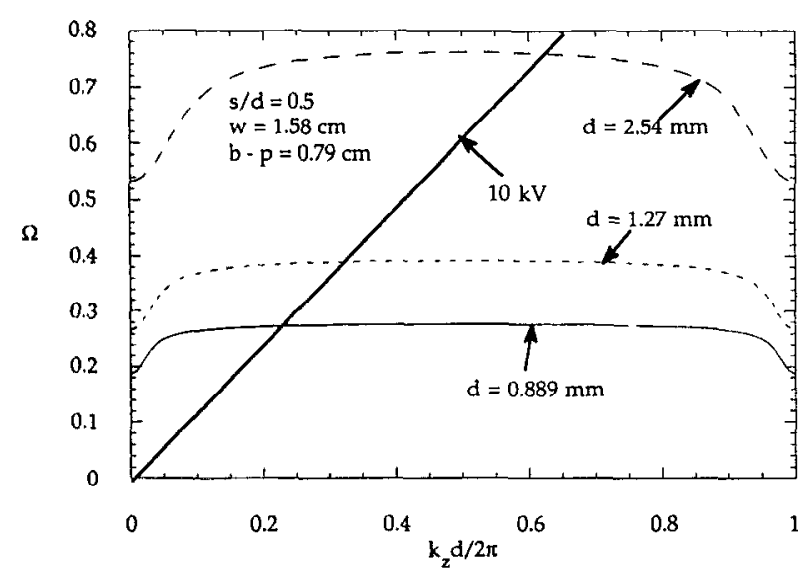

FIG. 3. Dispersion curves for the $\mathrm{TE}_{x 10}$ hybrid mode at various grating periods. shorter axial guide wave number $\left(k_{z} d\right)$ in the forward mode regime. Figure 3 illustrates that the values of $\Omega$ of the dispersion curve are reduced as the grating period $d$ is reduced at constant groove depth. The intersection between the $10 \mathrm{kV}$ beamline and the dispersion curve shifts further into the forward wave mode regime as the grating period is reduced. Therefore, to operate in the forward wave mode regime with a low beam energy, the rectangular grating amplifier requires short periods and deep groove gratings. ${ }^{17}$

The linear growth rates in the forward wave mode regime are also calculated. A code was written incorporating a finite axial thermal velocity spread for the beam that we have named GRAMP (GRating AMPlifier). This code can solve complex roots for a complex dispersion relation and generate contour plots. The complex dispersion relation in Eq. (16) is evaluated exactly utilizing GRAMP to obtain the linear growth rate.

There are two root finders utilized in the program. Muller's method is utilized to obtain the complex roots in the complex dispersion relation and the bisection method is utilized to obtain the roots of the vacuum dispersion relation. Wave growth occurs in the neighborhood of the intersection between the beamline and the grating dispersion curve (resonance point) in the vacuum dispersion relation. At this point the phase velocity of the slow wave is equal to the electron velocity. The amplification of the electromagnetic slow wave occurs when the phase velocity of the slow wave is slightly lower than the electron's velocity. The wave growth in the forward wave mode regime is a convective (spatial) growth, which is desired for an amplifier system.

\section{B. Illustrative examples}

To quantitatively illustrate various physical phenomena described in the theoretical analysis, a particular set of parameters (inspired by a design for future experimental verification) is selected. The illustrative case includes the specific values of $d=0.889 \mathrm{~mm}, s=0.445 \mathrm{~mm}, w=1.58 \mathrm{~cm}$, $p=0.66 \mathrm{~cm}$, and $b=1.45 \mathrm{~cm}$. The sheet electron beam thickness $t$ is $2 \mathrm{~mm}$ and the current density $J$ is $0.8 \mathrm{~A} / \mathrm{cm}^{2}$. These parameters are utilized for the growth rate calculations below. Figure 4 displays the dispersion relation for the fundamental and higher-order hybrid modes. The cutoff frequency of the hybrid mode $\mathrm{TE}_{x l m}, l=1,2, \ldots$, $m=0,1,2, \ldots$, depends on the width of the waveguide if $l \neq 0$ and the height $b$ if $m \neq 0$. Therefore, by decreasing $b$ the cutoff frequency of the $T E_{x 10}$ mode will remain the same, but those for $\mathrm{TE}_{x 11}$ and $\mathrm{TE}_{x 12}$ modes will increase. This property can be used to create a waveguide that guarantees a single-mode propagation in a rectangular grating.

Figure 5 presents the normalized linear spatial growth rates $\kappa_{i}\left[\kappa_{i}=\operatorname{Im}\left(k_{z} d / 2 \pi\right)\right]$ for the $\mathrm{TE}_{x 10}$ and $\mathrm{TE}_{x 11}$ modes as a function of beam energy for the forward wave mode regime. The separation distance, $\delta$, between the grating surface and the sheet beam is chosen to be $0.5 \mathrm{~mm}$. The sheet beam is initially assumed to be monoenergetic with $v_{\mathrm{th}} / v_{0}=0 \%$. The growth rates for these two modes are comparable. In an amplifier, it is desirable to have only one 


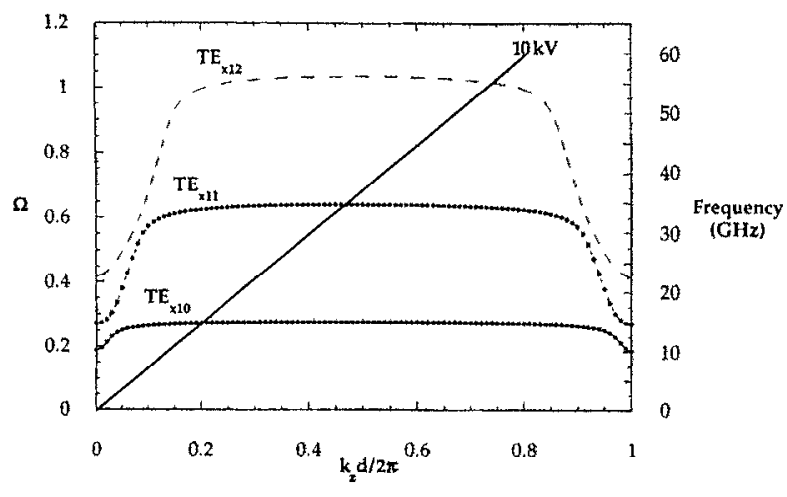

FIG. 4. Hybrid mode dispersion relation in the rectangular grating waveguide.

dominant growing mode. Therefore, the $\mathrm{TE}_{x 11}$ mode must be suppressed to avoid mode competition with the fundamental $\mathrm{TE}_{x 10}$ hybrid mode. The linear growth rates for the $\mathrm{TE}_{x 10}$ and $\mathrm{TE}_{x 11}$ modes versus $v_{\mathrm{th}} / v_{0}$ are shown in Fig. 6 . The beam energy for this case is chosen to be $20 \mathrm{kV}$ and $\delta=0.5 \mathrm{~mm}$. The $\mathrm{TE}_{x 11}$ mode linear growth rate decreases faster than that of the $\mathrm{TE}_{x 10}$ mode as $v_{\mathrm{th}} / v_{0}$ increases. For example, at $v_{\mathrm{th}} / v_{0}=5 \%$, the $\mathrm{TE}_{x 10}$ mode linear growth rate is only reduced by a factor of $\frac{1}{3}$ while that of the $T E_{x 11}$ mode is reduced by an order of magnitude. For the same beam energy, the axial wave number, $k_{z}$, for the $\mathrm{TE}_{x 11}$ mode is almost twice as large as that of the $\mathrm{TE}_{x 10}$ mode (see Fig. 4). The coupling, which depends on $\epsilon_{r n}$ in Eq. (4), between the beam and $T E_{x 11}$ mode, therefore, is weaker compared to that of $\mathrm{TE}_{x 10}$ mode. Hence, the growth rate for the $\mathrm{TE}_{x 11}$ mode is reduced and decreases faster as $v_{\text {th }}$ is increased, since $\epsilon_{r n}$ varies as $1 / k_{z}^{2}$.

Figure 7 displays a plot of the growth rate versus separation distance $\delta$. The beam energy is fixed at $20 \mathrm{kV}$ and $v_{\text {th }} / v_{0}=3 \%$. The axial electric field of the $\mathrm{TE}_{x 11}$ mode decays faster away from the grating surface compared to that of the $\mathrm{TE}_{x 10}$ mode. Hence, as shown in Fig. 7, the growth rate of the $\mathrm{TE}_{x 11}$ mode decreases much faster than that of

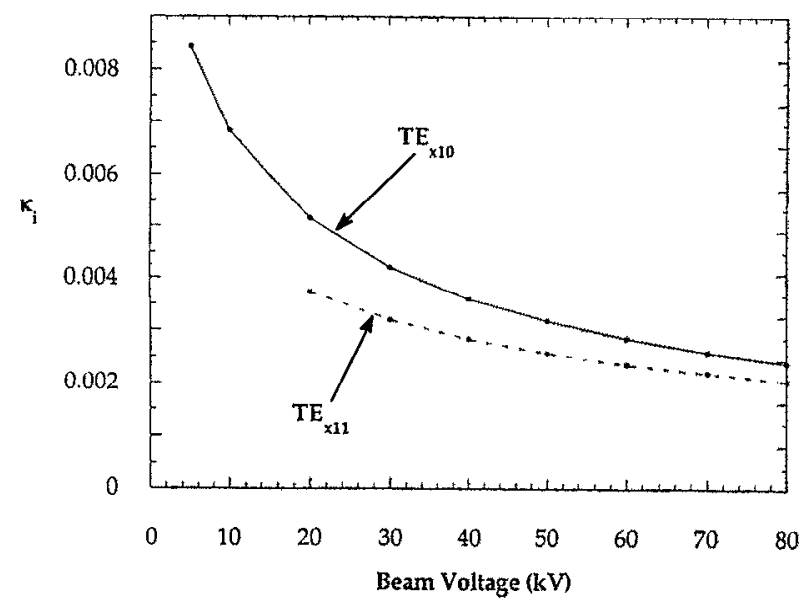

FIG. 5. The $\mathrm{TE}_{x 10}$ and $\mathrm{TE}_{x 11}$ modes linear growth rates at various energies of the monoenergetic sheet beam.

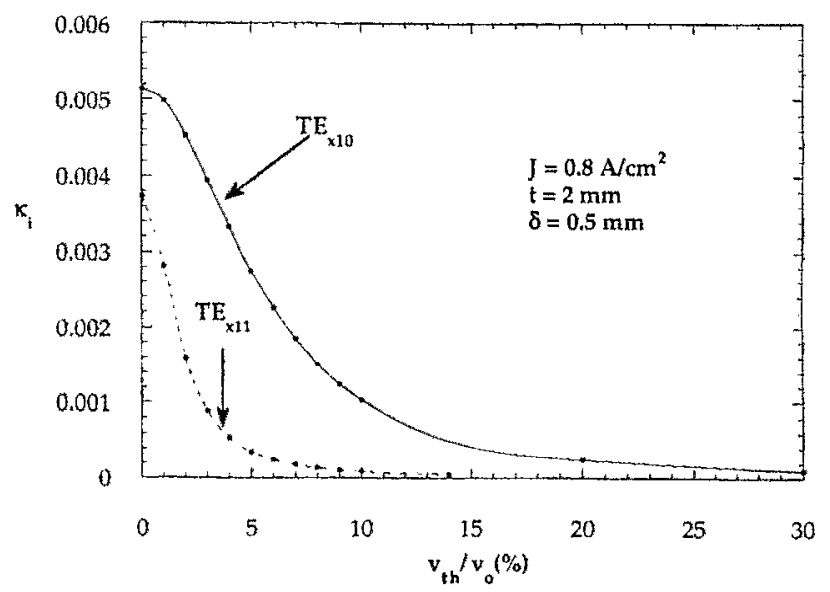

FIG. 6. Linear growth rates for $T E_{x 10}$ and $T E_{x 11}$ utilizing the sheet beam with nonzero axial thermal velocity spread.

the $T E_{x 10}$ as the beam-grating spacing is increased. The axial electric field and coupling with the electron sheet beam are both reduced, which yields a lower growth rate. Therefore, by introducing a few percent of axial thermal velocity spread (e.g., 2\%-3\%) and increasing the separation distance $\delta$ (e.g., $\delta=1 \mathrm{~mm}$ ), the $\mathrm{TE}_{x 11}$ mode linear growth rate can be preferentially and substantially reduced. At $v_{\text {th }} / v_{0}=3 \%$ and $\delta=1 \mathrm{~mm}$, the linear growth rate of the $\mathrm{TE}_{x 10}$ mode is an order of magnitude larger than that of the $T E_{x 11}$.

\section{ABSOLUTE AND CONVECTIVE INSTABILITY ANALYSIS FOR A ČERENKOV GRATING AMPLIFIER}

Briggs' criteria ${ }^{10}$ allows us to distinguish between absolute and convective instabilities by observing the behavior of the modes-i.e., the roots of the complex dispersion relation-in the complex $\omega$ and $k$ planes.

Briggs' criteria is a time asymptotic analysis for a localized point source in space for an infinitely long uniform system. In the criteria, the Laplace contour in the complex $\omega$ plane is deformed from the upper-half complex $\omega$ plane

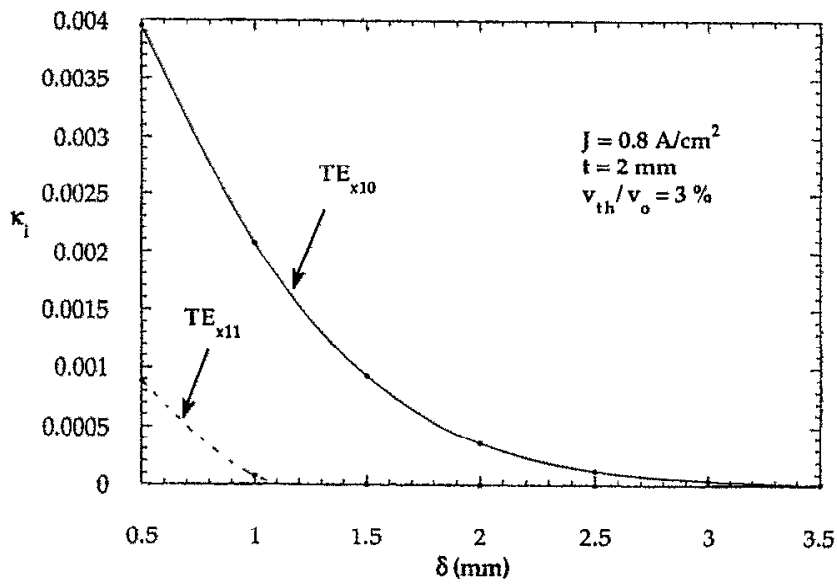

FIG. 7. A plot of linear growth rates for $T E_{x 10}$ and $T E_{x 11}$ versus the separation distance $\delta$. 


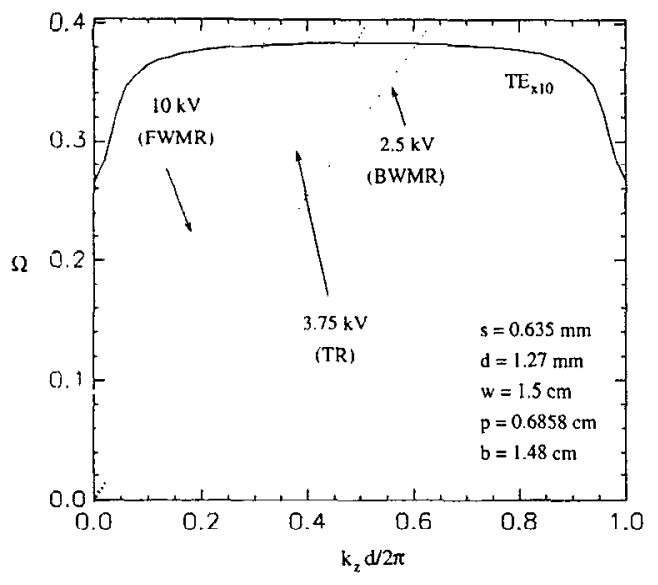

FWMR = Forward Wave Mode Regime BWMR = Backward Wave Mode Regime $\mathrm{TR}=$ Transition Regime

FIG. 8. The vacuum dispersion relation of the grating superposed with "beam synchronism lines."

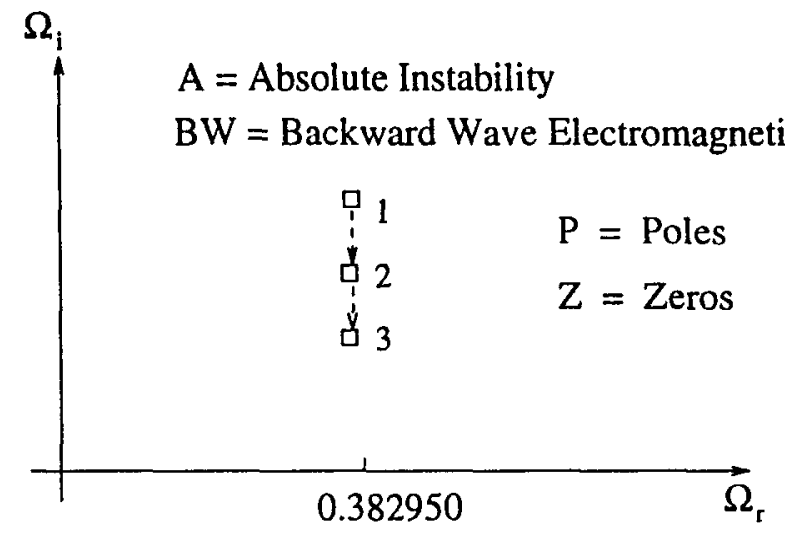

(a)

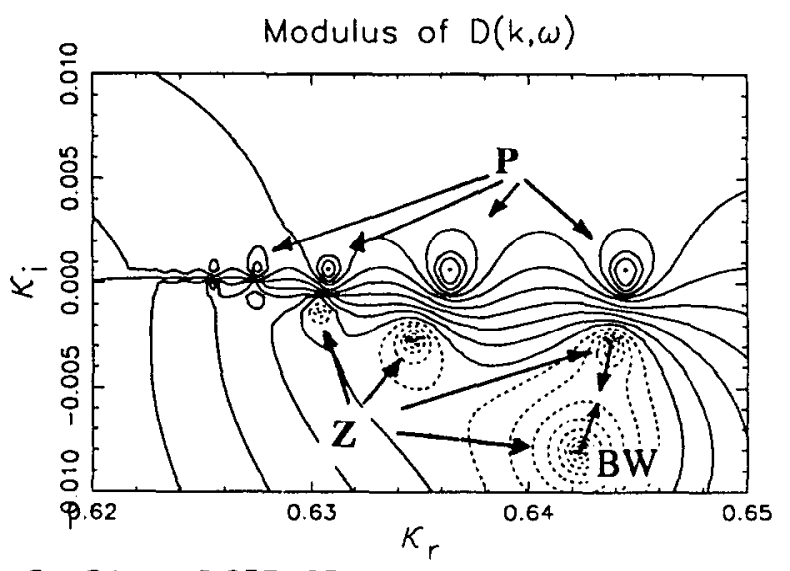

$\Omega_{i} \odot=0.25 \mathrm{E}-03$

(b)

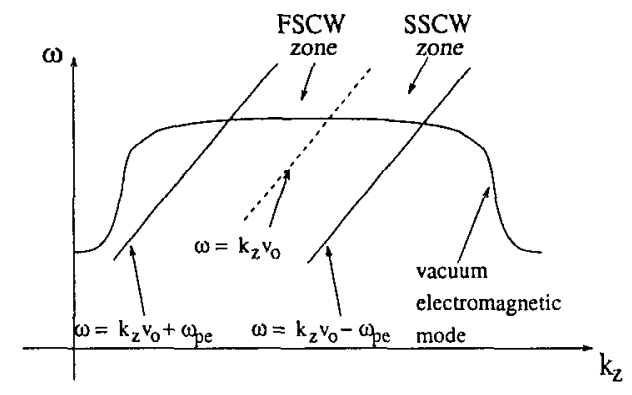

FIG. 9. Illustration of "slow space-charge wave" (SSCW) and "fast space-charge wave" (FSCW) zones.

$\left(\omega_{i}>0\right)$ to the real axis. In deforming the contour, the trajectories of the roots of $|D(\omega, k)|$ are observed in the complex $k$ plane. The deformation process means changing localized signal response from a transient one to steadystate response (time asymptotic response). According to the criteria, if one or more roots in the complex $k$ plane crosses the real $k$ axis in the process of the Laplace contour deformation, then these roots correspond to a convectively unstable root. This contour integration in the complex $k$ plane after the deformation will yield a finite value. This

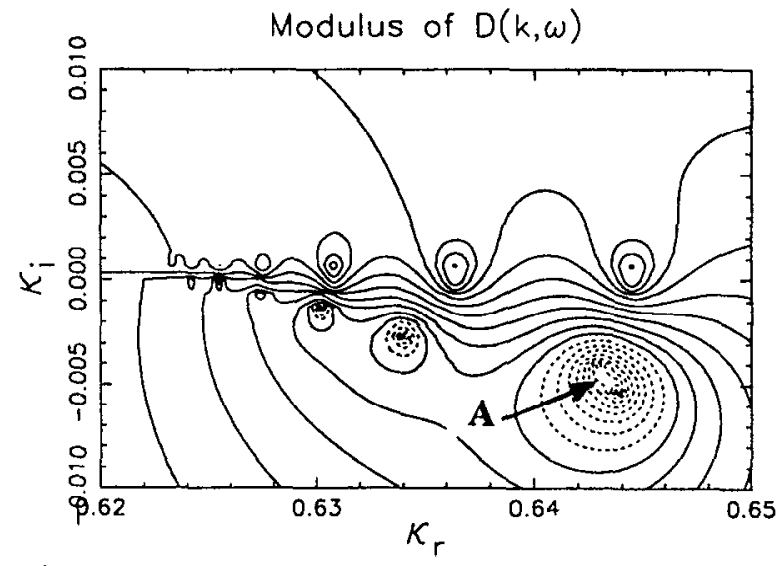

$\Omega_{\mathrm{i}} @ 2=0.23 \mathrm{E}-0.3$

(c)

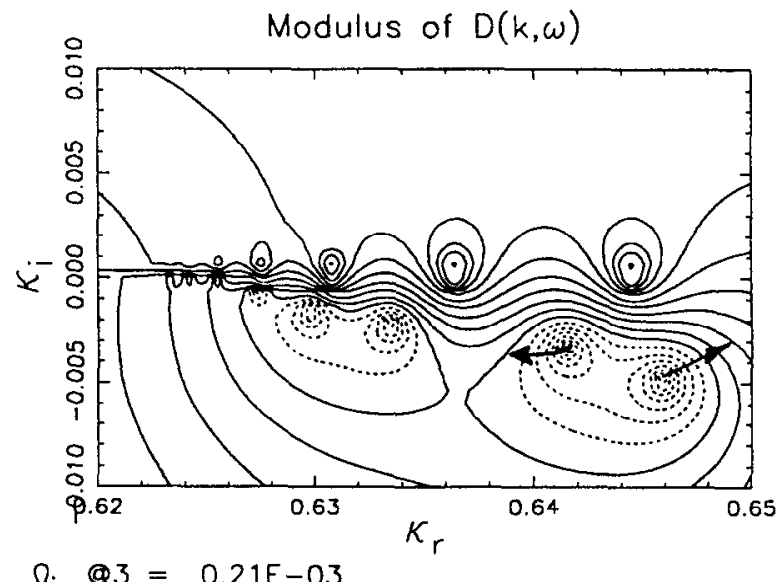

(d)

FIG. 10. The roots of $|D(\omega, k)|$ 's motion in the complex $\kappa$ plane, as $\Omega_{i}$ is varied at fixed $\Omega_{r}$ for backward wave operation with a monoenergetic sheet beam. 


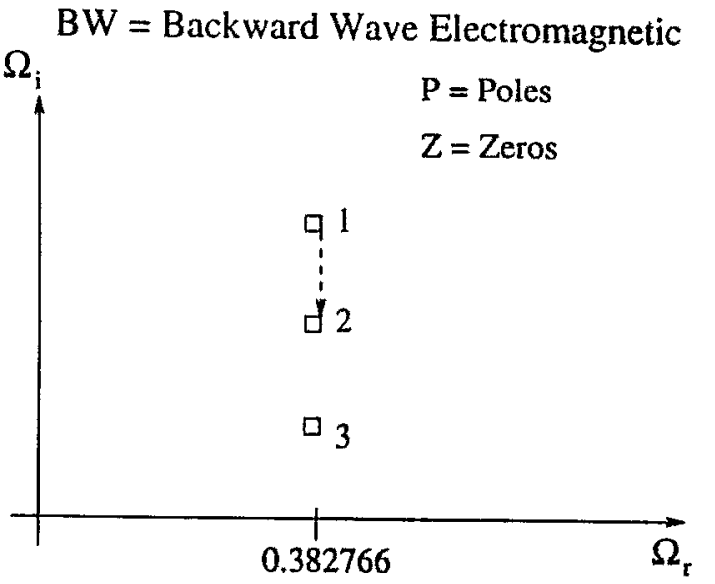

(a)

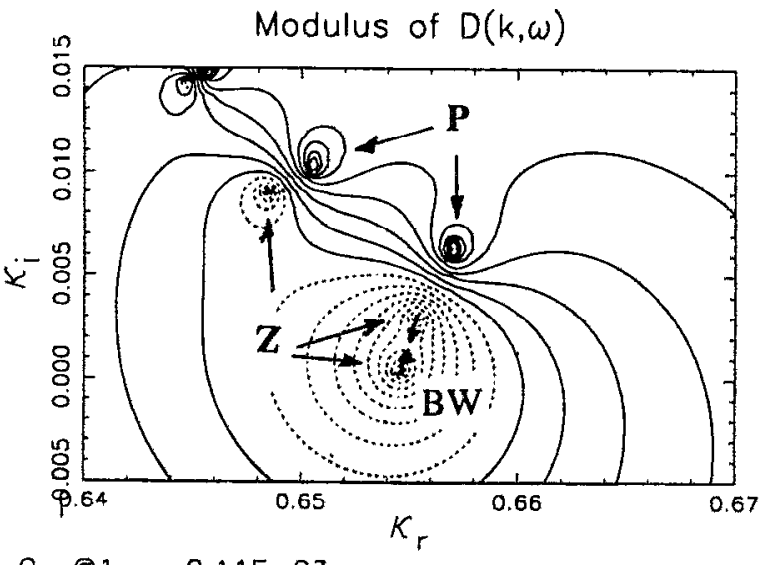

(b)

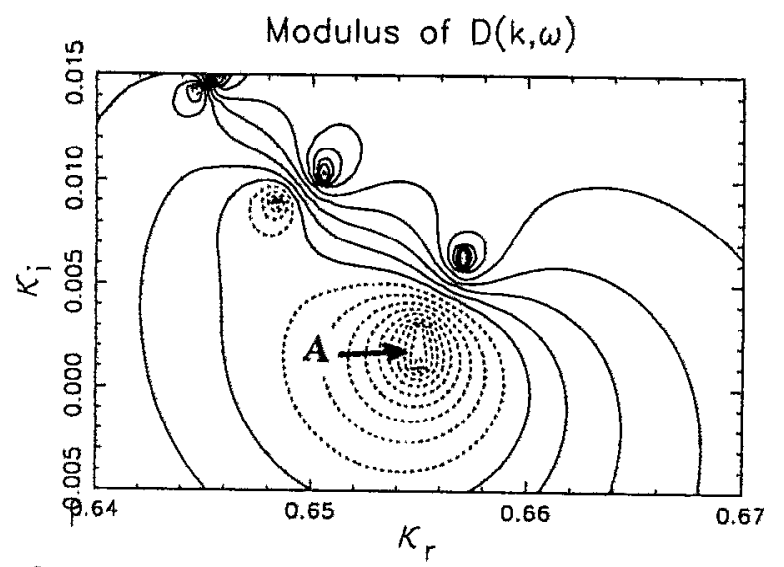

(c)

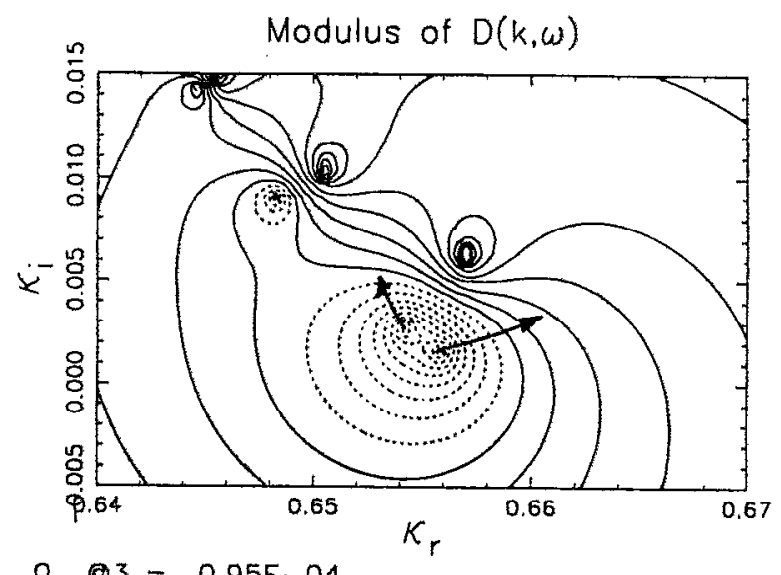

(d)

FIG. 11. The first absolute instabilities in the $3 \%$ axial thermal velocity spread case for backward wave operation.

means that for a convective instability, the amplitude of a signal at fixed point in space reaches a finite amplitude in steady state (i.e., the signal locally saturates). The absolute instability occurs, correspondingly, when Laplace contour deformation is prevented at some $\omega_{i}>0$ by the pinching of two roots from different halves of the complex $k$ plane (pole pinching). The point where the two roots pinch is called a saddle point. The complex $k$ contour integration is singular at that particular $\omega$ and asymptotically varies as $t^{-1 / 2} \exp \left(\omega_{i} t\right)$. This means that the signal amplitude grows indefinitely in time at every point in space and never saturates.

We apply the criteria to the interaction between the slow wave and the electron sheet beam propagating over the grating. In conceiving a grating amplifier system, we must understand the physical conditions that support convective wave growth. We utilized GRAMP to study absolute and convective instabilities for the slow-wave-sheet beam grating system. Given a value of $\omega$, the code locates the roots of $|D(\omega, k)|$ in the complex $k$ plane. By fixing $\omega_{r}$ and varying $\omega_{i}$, the trajectory of the roots in the complex $k$ plane can be followed. They are traced to determine whether they are spatially or temporally unstable accord- ing to Briggs' criteria. In the analysis, we have included the effects of an axial thermal beam spread on wave growth.

In the limit of a monoenergetic beam, when $v_{\text {th }} \rightarrow 0$, the first derivative of the plasma dispersion function in the complex dispersion function becomes

$$
\lim _{v_{\mathrm{th} \rightarrow 0}} Z^{\prime}=\frac{k_{z n}^{2} v_{\mathrm{th}}^{2}}{\left(\omega-k_{z n} v_{0}\right)^{2}} .
$$

As an illustration, a grating is considered with a periodicity of $d=0.127 \mathrm{~cm}$, a groove depth $p=0.6858 \mathrm{~cm}$, and width $v=1.58 \mathrm{~cm}$. The clearance from the grating surface to the top conductor is $0.79 \mathrm{~cm}$. The sheet-beam thickness is $1 \mathrm{~mm}$ and the current density is $1.68 \mathrm{~A} / \mathrm{cm}^{2}$. The distance from the center of the beam to the grating surface is $1 \mathrm{~mm}$. The vacuum dispersion relation is shown superposed with several "beam synchronism lines" satisfying $\omega=k_{z} y_{0}$ in Fig. 8. These beamlines exemplify different regimes of interaction between the electron beam and the electromagnetic wave. These regimes are categorized as the forward wave mode, backward wave mode, and transition regimes, as discussed below. 


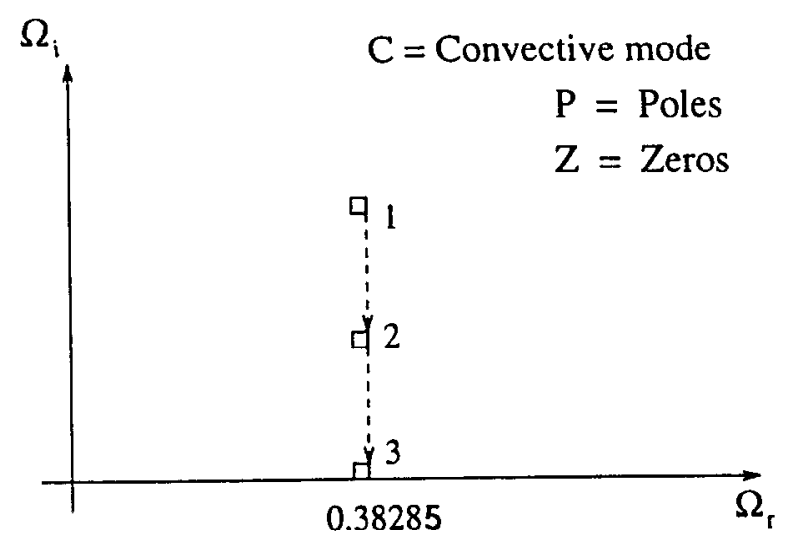

(a)

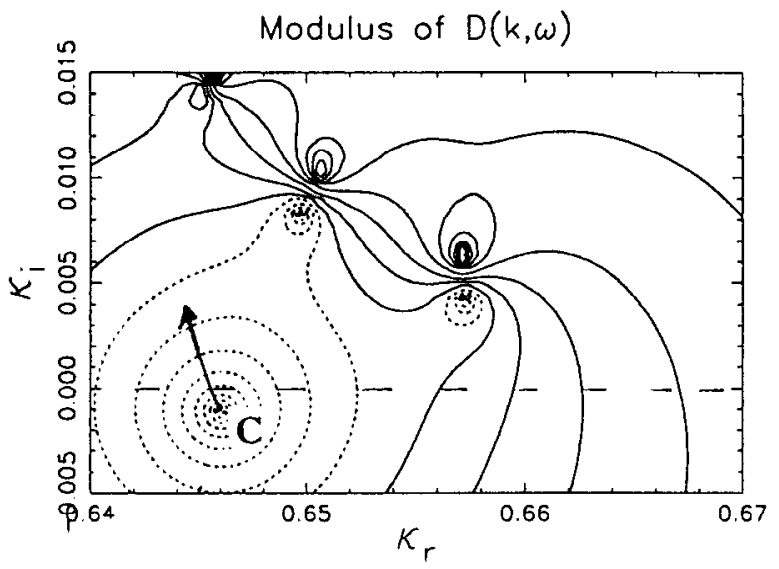

$\Omega_{i} \quad 1=0.12 \mathrm{E}-03$

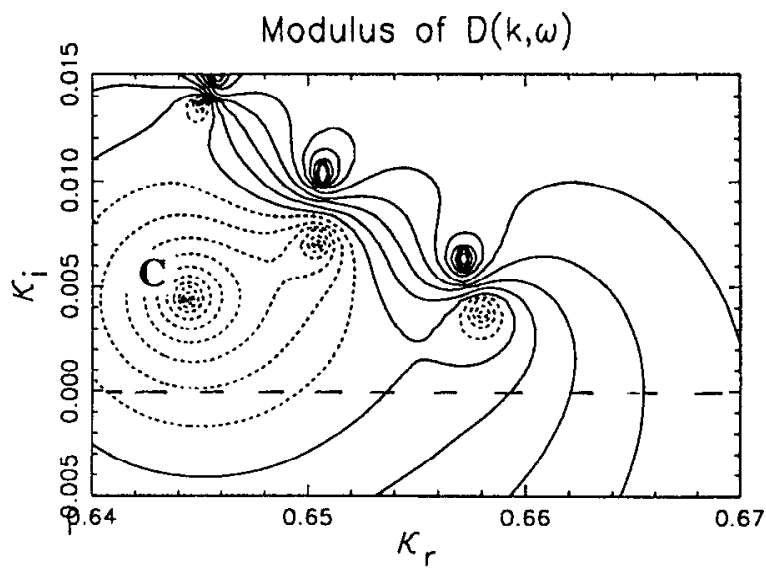

$\Omega_{i} @ 2=0.60 \mathrm{E}-04$

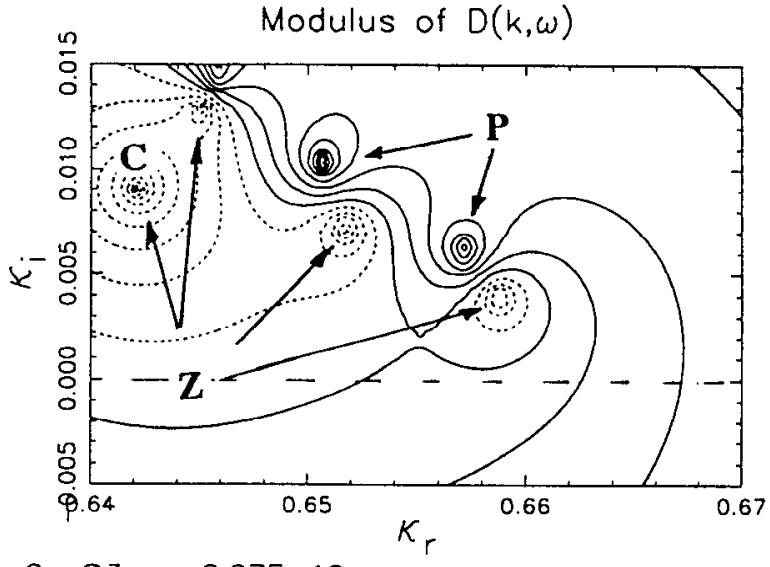

(d)

FIG. 12. The convective mode in the $3 \%$ axial thermal velocity spread for backward wave opcration.

Before moving on to a more specific discussion, we note that with nonzero, finite thickness beams in a bounded waveguide there are an infinite number of propagating space-charge normal modes on the beam. ${ }^{18}$ For purposes of a subsequent discussion, we will identify any beam spacecharge waves with frequencies $k_{z} v_{0}-\omega_{p e}<\omega<k_{z} v_{0}$ as belonging to the "slow space-charge wave" (SSCW) zone, while those space-charge waves found in the range $k_{z} v_{0}<\omega<k_{z} v_{0}+\omega_{p e}$ are identified as belonging to the "fast space-charge wave" (FSCW) zone. An exaggerated illustration of this is displayed in Fig. 9.

\section{A. Backward wave mode operation}

The backward wave mode operation is divided into two cases: a backward electromagnetic wave interacting with a monoenergetic sheet beam and a sheet beam with nonzero axial thermal velocity spread, respectively.

\section{Monoenergetic sheet beam case}

We consider a $2.5 \mathrm{kV}$ monoenergetic electron sheet beam streaming over the grating structure. At this beam energy level, the slow-wave and electron beam interactions occur in the backward wave mode regime, as shown in Fig. 8. In the backward wave mode regime, the electromagnetic wave phase velocity is in the same direction as the beam velocity, but its group velocity is in the opposite direction to the beam velocity. To be able to justify the presence of either absolute or convective instabilities for the slow-wave and electron beam interaction, we need to fix $\omega_{r}$ and vary $\omega_{i}$ in the complex $\omega$ plane and observe the trajectory of the zeros of $|D(\omega, k)|$ from Eq. (16) in the complex $k$ plane.

Let $\Omega=\Omega_{r}+j \Omega_{i}$ be the normalized complex frequency where $\Omega=\omega d / c, \Omega_{r}=\omega, d / c$ and $\Omega_{i}=\omega_{i} d / c$, and $\omega_{r}$ and $\omega_{i}$ are the real and imaginary parts of $\omega$, respectively. Let $\kappa=\kappa_{r}+j \kappa_{i}$ be normalized complex wave number, where $\kappa=k d / 2 \pi, \kappa_{r}=\operatorname{Re}\left(k_{z} d / 2 \pi\right)$, and $\kappa_{i}=\operatorname{Im}\left(k_{z} d / 2 \pi\right)$. Figure 10 shows the zeros of $|D(\omega, k)|$ that correspond to different modes in the complex $\kappa$ plane as $\Omega_{i}$ is varied. According to the criteria, the process must be repeated for all $\Omega_{r}$. The series of zeros close to $\kappa_{i}=0$ corrcspond to the slow space-charge modes. Below these modes is the slow-wave backward electromagnetic mode, as shown in the lower left corner of Fig. 10. In Fig. 10, the first absolute instability in the system is observed at $\Omega=0.382950+j 0.00023$. As $\Omega_{i}$ is varied from point (1) to point ( 3 ) in the complex $\Omega$ plane [Fig. 10(a)] at fixed $\Omega_{r}=0.382950$, the roots of $|D(\omega, k)|$ in the complex $\kappa$ plane migrate [Figs. 10(b)$10(\mathrm{~d})$ ]. As $\Omega_{i}$ is moved from point (1) to (2), the back- 


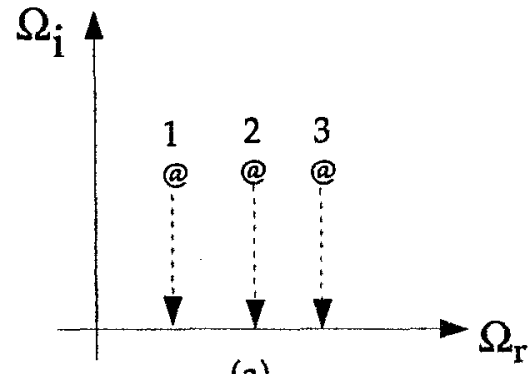

(a)

$$
\begin{aligned}
& \text { @ - zeros in } \Omega \text { plane } \\
& \text { \# - slow space charge wave mode } \\
& \text { * - backward electromagnetic mode }
\end{aligned}
$$

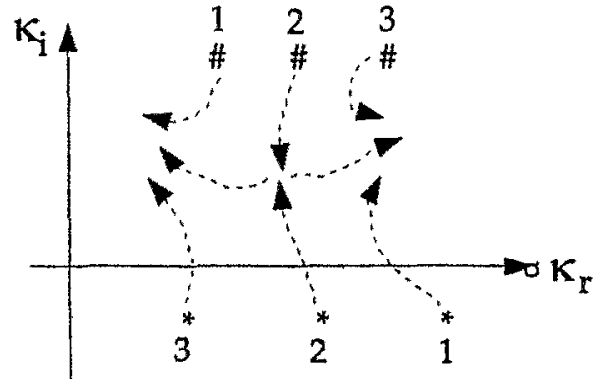

(b)

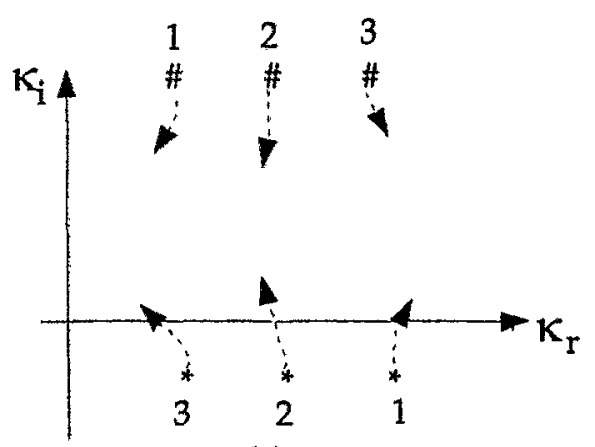

(c)

FIG. 13. Trajectory of the zeros in complex $\kappa$ plane for small thermal spread (b) and large thermal spread (c) as the Laplace contour in the complex $\Omega$ plane [shown in (a)] is deformed.

ward wave electromagnetic mode from the lower-half complex $\kappa$ plane coalesces with a slow space-charge modes that originates from the upper half of the complex $\kappa$ plane. The saddle point occurs at $\Omega_{i}=0,00023$. This signifies the presence of an absolute instability. The modes split as $\Omega_{i}$ is moved from point (2) to (3) [Fig. 10(d)]. By repeating the same method at $\Omega_{r}=0.382959$, we can find another absolute instability at $\Omega_{i}=9 \times 10^{-5}$ associated with the coupling between the slow electromagnetic mode and a different slow space-charge mode. A series of absolute instabilities can be found by the same method described above at other discreet values of $\Omega_{r}$. However, the corresponding values of $\Omega_{i}$ found are less than that of the first one. In a system with a large number of absolute instabilities, the temporal growth is dominated by the largest $\Omega_{i}$ in the system as $t \rightarrow \infty$, which in this case occurs at $\Omega_{r}=0.382950$ with $\Omega_{i}=0.00023$. In the monoenergetic case, no convective instability is observed, since there are no zeros that cross the real $\kappa$ axis, as the Laplace contour is deformed to the real $\Omega$ axis.

\section{Nonzero axial thermal velocity spread effects}

We now introduce a nonzero axial velocity spread. The electron beam is assumed to have a Maxwellian distribution for its axial velocity. Figure 11 shows the zeros of $|D(\omega, k)|$ in the complex $\kappa$ plane for the case of an axial thermal velocity spread of $3 \%$. The introduction of axial velocity spread distorts the positions of the zeros. In the monoenergetic case, all the slow space-charge modes lie close to each other, forming almost a straight line parallel to $\kappa_{i}=0$. In the nonzero axial velocity spread case, the zeros corresponding to the slow space-charge modes are pushed up and distributed in the upper half of the complex $\kappa$ plane. Varying $\Omega_{i}$ at fixed $\Omega_{r}$ in the complex $\Omega$ plane and repeating the procedure for all $\Omega_{r}$, we find only two absolute instabilities for this case. Figure 11 shows the occurrence of the first absolute instability. Figures $11(\mathrm{~b})-11$ (d) show the trajectory of the zeros in the complex $\kappa$ plane as $\Omega_{i}$ is varicd from point (1)-(3). When $\Omega_{i}$ is decreased from point (1)-(2), one of the zeros from the upper-half complex $\kappa$ plane pinches with a zero from the lower-half plane [Fig. 11(b)]. This occurs at $\Omega=0.382766$ $+j 1.0 \times 10^{-4}$. Then these two zeros split as $\Omega_{i}$ varied from point (2)-(3). The second absolute instability is found at $\Omega=0.382822+j 1.0 \times 10^{-5}$ by utilizing the same method as described above. Also note the introduction of thermal spread reduces $\Omega_{i}$, indicating a smaller temporal growth. The absolute instability also occurs at a lower $\Omega_{r}$ compared to that for the monoenergetic case. Hence, the thermal velocity spread shifts the point of maximum absolute unstable growth to a lower frequency.

In addition to the absolute instabilities, the introduction of a small axial thermal velocity allows the interaction to support convective wave growth in this regime. Figure 12 shows an example occurring in the SSCW zone. As $\Omega_{i}$ is reduced to zero [Fig. 12(a)], one of the roots of $|D(\omega, k)|$ crosses the real $\kappa$ axis from the lower half to the upper half of the complex $\kappa$ plane [Figs. 12(b)-12(d)], which indicates a convective root in the $-z$ direction. This root corresponds to the backward electromagnetic mode 
whose phase velocity is in the $+z$ direction and group velocity in the $-z$ direction. In the FSCW zone, the backward wave electromagnetic mode always decays, as expected.

In general, when the thermal velocity spread is small ( $<4 \%$ in this example), the space-charge modes are close to the $\kappa_{r}$ axis in the complex $\kappa$ plane. Also, the backward wave electromagnetic mode crosses the $\kappa_{r}$ axis and penetrates deeply into the upper-half $\kappa$ plane from the lowerhalf $\kappa$ plane [Fig. 13(b)], as the Laplace contour is deformed in the complex $\Omega$ plane [Fig. 13(a)]. These two features of low thermal velocity spread enable the backward wave electromagnetic mode to coalesce with a slow space-charge mode in the deformation process. Hence, an absolute instability exists in this condition.

However, if the thermal velocity spread is increased further ( $>4 \%$ in this particular example), no absolute instability is found. Only convective mode growth persists. For this case, the space-charge modes are shifted farther away from the $\kappa_{r}$ axis into the upper-half $\kappa$ plane. Moreover, the backward wave electromagnetic mode does not cross the $\kappa_{r}$ axis as deeply into the upper-half $\kappa$ plane during the deformation process. Hence, the backward wave electromagnetic mode is never observed to coalesce with a slow space-charge mode [Fig. 13(c)]. The thermal velocity spread has caused the phase of the waves that propagate in the $+z$ and $-z$ direction not to interfere constructively. Even though the wave is amplified in the $-z$ direction, there is not enough distributed positive feedback for this wave to produce an absolute instability.

Figure 14 shows the peak convective growth rate and instantaneous bandwidth for various axial velocity spreads in the backward wave mode regime. Note that the plot starts at $v_{\mathrm{th}} / v_{z}=4 \%$, since the absolute instability is still present when $v_{\mathrm{th}} / v_{z}<4 \%$. As the thermal spread increases, the convective wave growth decreases, but the bandwidth increases in a manner reminiscent of a finite-timetransform limited gain process. The product of the gain and bandwidth decreases, but only rather slowly. This regime may be useful for backward wave amplifier applications.

When the axial thermal velocity spread is increased further, i.e., up to $15 \%$, no instabilities are observed. In this case the average energy transfer from the electron beam to the electromagnetic wave is less than or equal to the energy transfer from the electromagnetic wave to the beam via Landau damping.

\section{B. Forward wave mode regime}

If the beam energy is increased to $10 \mathrm{kV}$ for the same grating parameters, the slow-wave and electron beam interaction occurs in the forward wave mode regime. This is illustrated in Fig. 8. Unlike the backward wave mode case, the forward slow-wave electromagnetic mode is difficult to identify near the resonance region because it lies close to other space-charge modes. In the neighborhood of the resonance region, we can identify the amplifying wave, the decaying wave, and the oscillating modes. Wave growth is only found in the SSCW zone. In the forward wave mode

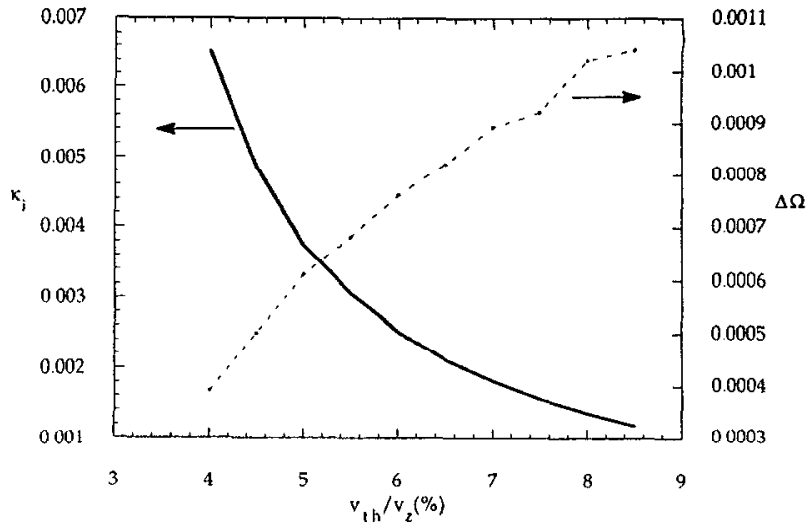

(a)

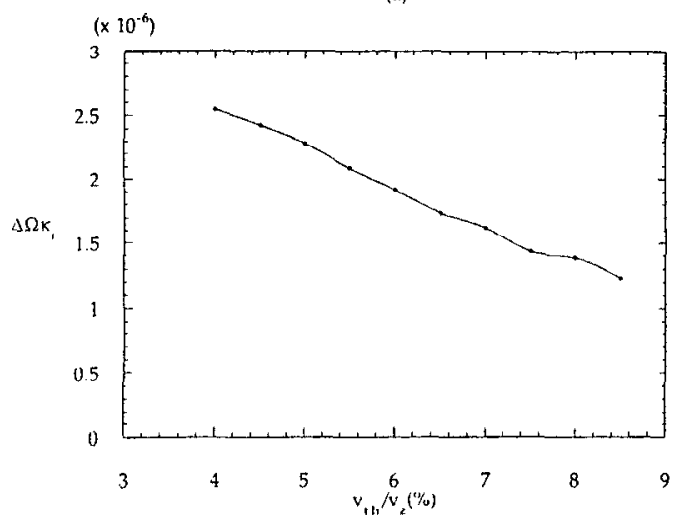

(b)

FIG. 14. (a) Convective wave growth and bandwidth at various thermal spread in the backward wave mode regime. (b) The product of wave growth and bandwidth.

regime, no absolute instabilities are observed, even for a monoenergetic beam. In this regime, all of the normal modes (space charge and electromagnetic) propagate in the $+z$ direction. Since there is no $-z$ direction propagating wave (unless there is a reflection at some point in $z$ ), no constructive interference necessary for an absolute instability is possible.

Figure 15 shows the convective mode for a $10 \mathrm{kV}$ beam with a $3 \%$ axial thermal velocity spread. In the SSCW zone, as $\Omega_{i}$ is reduced to zero [Fig. 15(a)], the zeros of $|D(\omega, k)|$ in the upper-half $\kappa$ plane cross the real $\kappa$ axis to the lower-half plane [Figs. 15(b)-15(d)], which signify the presence of an amplifying wave in the $+z$ direction. In the FSCW zone, the forward wave electromagnetic mode decays. The phase and group velocities for this mode are in the $+z$ direction.

Figure 16 presents the convective wave growth and bandwidth for various axial thermal sprcads for the forward wave mode regime. In this regime, both growth rate and bandwidth decrease when the thermal spread increases. The product of growth rate and bandwidth therefore also decreases (rather rapidly) as $v_{\mathrm{th}} / v_{\mathrm{z}}$ increases. The convective growth becomes negligible if the axial thermal velocity spread is large, i.e., $15 \%$. 


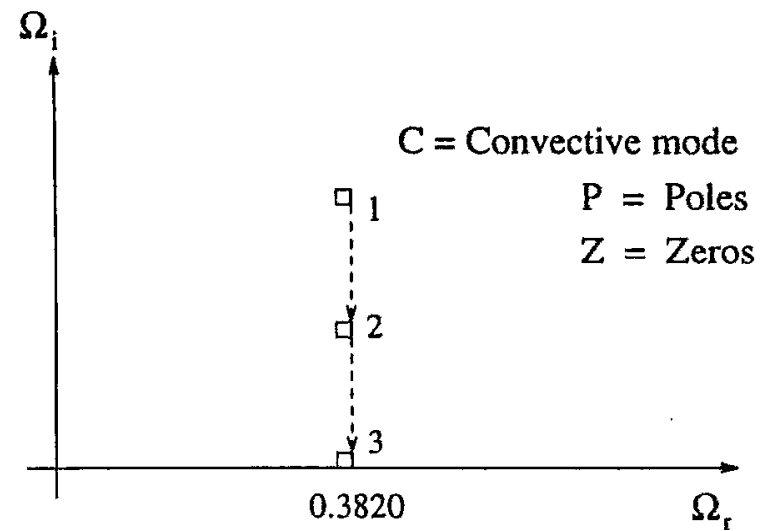

(a)

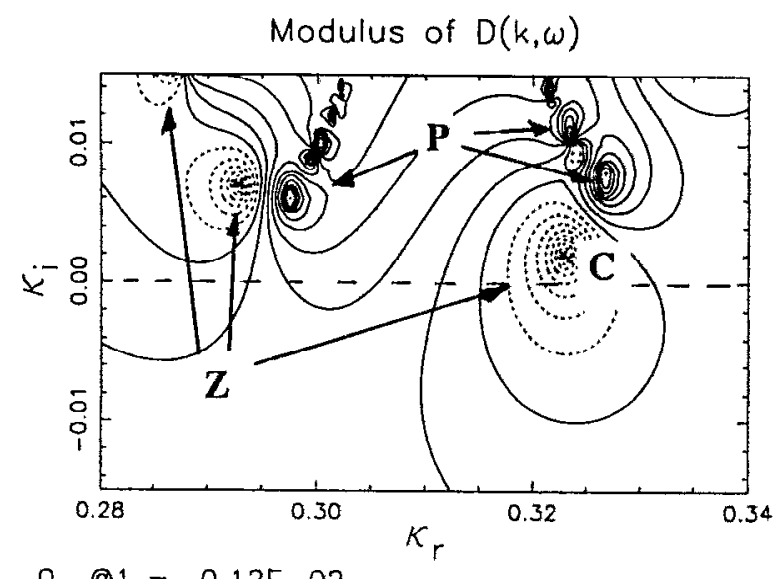

$\Omega_{\mathrm{i}} @ 1=0.12 \mathrm{E}-02$

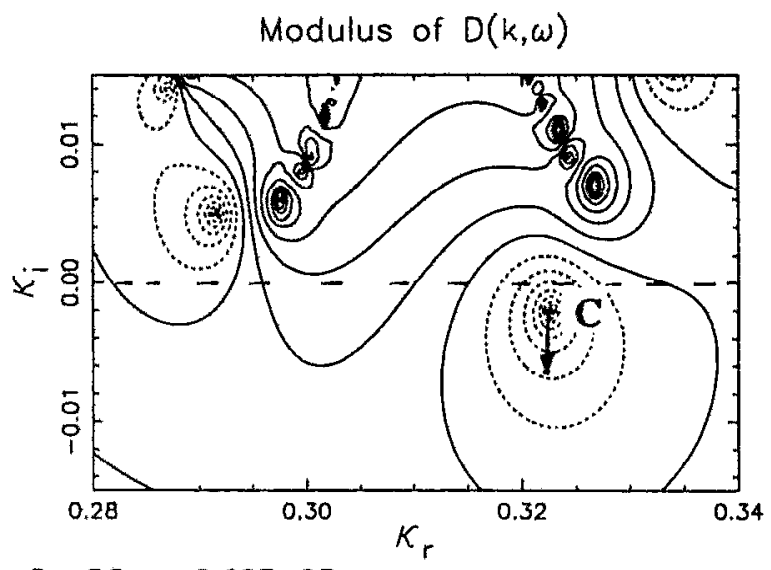

(c)

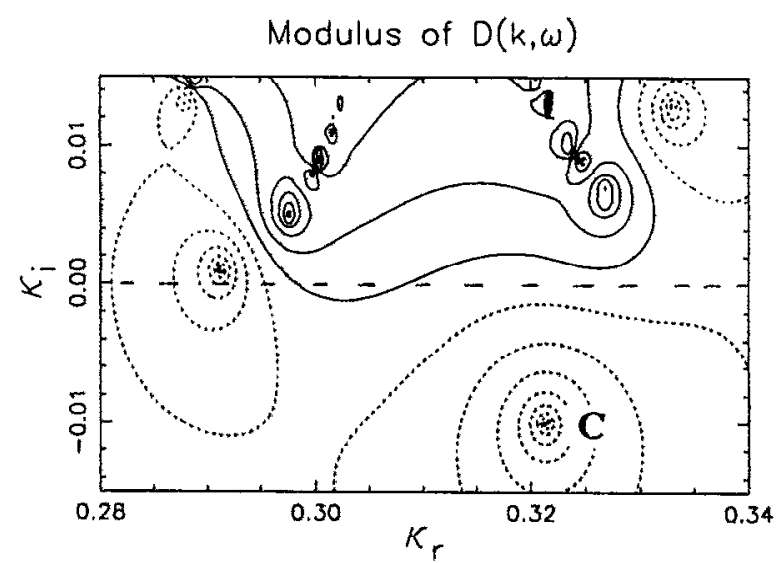

$\Omega_{i}$ (3) $3=-0.22 E-18$

(b)

(d)

FIG. 15. The amplifying mode for the forward mode regime with a $3 \%$ axial thermal velocity spread.

\section{Transition regime}

When one or morc fast space-charge modes lie in the forward wave mode regime $(k, d / 2 \pi<0.5)$ and one or more slow space-charge modes lie in the backward wave mode regime $\left(k_{z} d / 2 \pi>0.5\right)$, the interaction is defined as operating in the transition regime. The $3.75 \mathrm{kV}$ electron beam is in this regime, for example, as shown in Fig. 8. For a monoenergetic beam at $3.75 \mathrm{kV}$, a few slow space-charge modes lie in the backward wave mode regime and the rest of the space-charge modes lie in the forward wave mode regime. In this region, whether the beam is monoenergetic or not, both absolute and convective instabilities are always present, provided the Landau damping does not dominate the interaction. Both forward and backward waves are amplified. If the phases of two such counterpropagating waves happen to interfere constructively, the instability becomes absolute. However, if the phase does not interfere constructively, the instability remains convective. In this regime, usually the instability is convective at lower frequencies and terminates as an absolute instability at a higher frequency (for fixed beam voltage). Hence, one can expect the experimentally observed growth in the transition regime to always manifest itself as an absolute instability.
Figure 17 shows the unstable frequency range for a monoenergetic sheet beam at $3.75 \mathrm{kV}$. The convective growth in the transition regime is higher than that for the forward and backward wave mode regimes. This is due to the fact that the axial electric field close to the grating surface is stronger in this region, hence the coupling between the electron beam and the electric field is higher, which yields a higher convective growth rate for the electromagnetic wave.

To reiterate, an absolute instability occurs only when there are at least two modes with different group velocity directions. In the forward wave mode regime, all modes have a group velocity in the $+z$ direction, therefore no absolute instability is observed in this regime. However, in the transition and backward wave mode regimes, modes with opposite group velocity directions coexist, so that absolute instabilities are possible.

For example, in the backward wave mode regime, the absolute instabilities arise from constructive interference between a forward propagating slow space-charge wave (SSCW) and a backward electromagnetic (EM) wave. This interference can be suppressed by introducing a large 

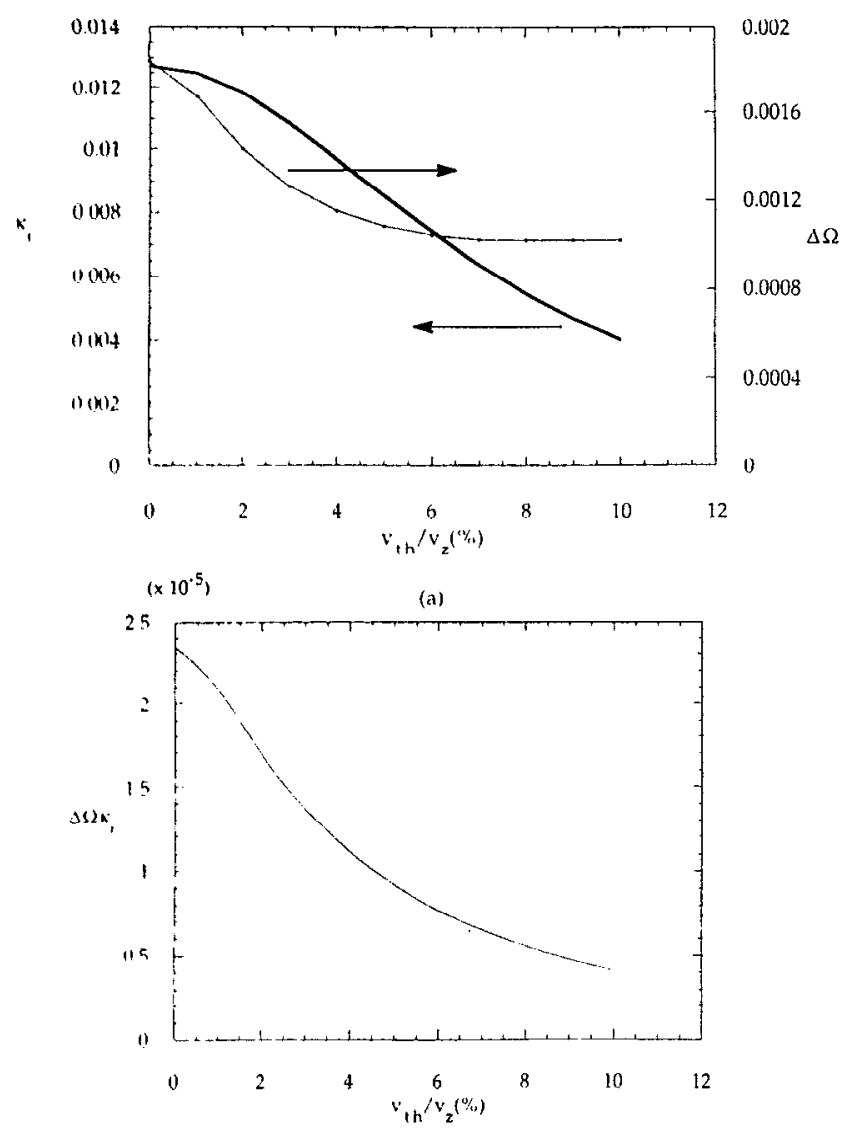

(b)

FIG. 16. (a) Convective wave growth and bandwidth at various thermal spread in the forward wave mode regime. (b) The product of wave growth and bandwidth.

thermal spread to the electron beam, as shown in Fig. $13(c)$.

In the transition regime, however, absolute instabilities can arise from constructive interference between either a forward SSCW and backward EM wave or forward and backward EM modes. Constructive interference for the first combination can be suppressed by large thermal velocity spread. However, absolute growth due to the latter combination cannot be suppressed by velocity spread, unless so much velocity spread is introduced that all instabilities are eliminated.

\section{SUMMARY}

We have derived the complex dispersion relation for a sheet electron beam interacting with hybrid modes in a rectangular grating waveguide. We have also explored the choice of grating parameters that allow operation of a rectangular grating amplifier in the forward wave mode regime with a low-energy beam. A particular case with a deep groove (i.e., $p=6.6 \mathrm{~mm}$ ) and a short grating period (i.e., $d=0.889 \mathrm{~mm})$ is identified that allows the rectangular grating amplifier to amplify microwaves (i.e., $14 \mathrm{GHz}$ ) with a $10 \mathrm{kV}$ electron beam in the forward wave mode regime. To scale up the operating frequency of this device utilizing the same beam energy, the grating period must be

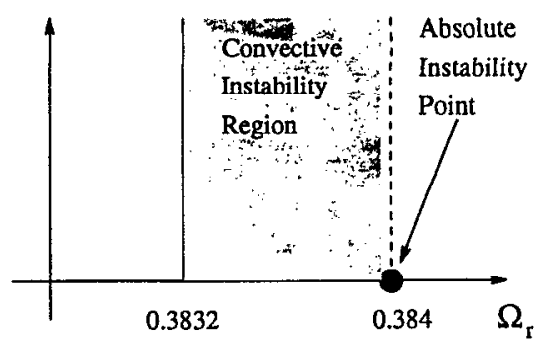

FIG. 17. Frequency range for absolute and convective instability in the transition regime for $3.75 \mathrm{kV}$ monoenergetic electron beam.

reduced. A large groove depth, $p$, causes the dispersion curves for the uncoupled dispersion relation to flatten. The axial electric field for this type of structure is confined very closely to the grating surface, and its amplitude decays exponentially away from the grating surface. Therefore, to yield high efficiency, the sheet electron beam must be aligned very closely to the grating surface, since the gain of this device is determined by the strength of the coupling between the clectron beam and the axial electric field.

The forward wave mode regime will allow amplifier operation. The linear growth rates of the $\mathrm{TE}_{x 10}$ and $\mathrm{TE}_{x 11}$ hybrid modes in this regime are also presented. In the case of a monoenergetic sheet beam that is close to the grating surface, the $T E_{x 11}$ mode will compete with the $T E_{x 10}$. However, the linear growth rate of the $\mathrm{TE}_{x 11}$ mode is reduced substantially by modest amounts of axial thermal velocity spread and by modest increases in the beam grating separation distance.

Three different regimes of operation are investigated utilizing the Briggs' criteria for absolute and convective wave growth. In the forward wave mode regime, the instability is always convective for both the monoenergetic beam and the beam with a thermal spread. In the transition regime, both convective and absolute instabilities are always present for both beam cases, and, hence, absolute instabilities are expected to dominate. In the backward wave mode regime, the instability is solely absolute for a monoenergetic beam, a mixture of absolute and convective with a small thermal velocity spread (e.g., $v_{\text {th }} / v_{0} \leqslant 4 \%$ ), and solely convective for a larger thermal spread beam (e.g., >4\%). An increase in thermal spread also shifts the mode with peak absolute growth to a slightly lower frequency. In general, for small thermal spread the growthbandwidth product is higher for the forward wave amplifier than for backward wave amplifier. However, the growth-bandwidth product is more sensitive to velocity spread in the forward than in the backward wave amplifier regime.

The backward wave amplifier operation regime is made possible by increasing the thermal spread to a moderate value. Of course, a consequence is that the peak growth rate is reduced. However, this regime of operation with a lower quality beam is of interest, since the linear growth rate of this device, even though it is reduced, is still significant.

Future work will include experimental investigation of 
absolute and convective wave growth in the three regimes mentioned above, and theoretical and experimental investigations of finite length effects and bandwidth enhancement methods for a Cerenkov amplifier.

\section{ACKNOWLEDGMENTS}

This work was supported in part by the Department of Defense Vacuum Electronics Initiative as managed by the Air Force Office of Scientific Research (Grant No. AFOSR-91-0381), and by a National Science Foundation Presidential Young Investigator Award (ECS-9057675).

'I. M. Frank and I. Tamm, Dokl. Akad. Nauk. 14, 109 (1937).

${ }^{2}$ J. G. Linhart, J. Appl. Phys. 26, 527 (1955).

${ }^{3}$ J. M. Wachtel, J. Appl. Phys. 50, 49 (1979).

${ }^{4}$ M. Botton and A. Ron, IEEE Trans. Plasma Sci. PS-16, 225 (1988).

${ }^{5}$ L. Schacter and A. Ron, Nucl. Instrum. Methods. Phys. Res. A 285, 262 (1989).

${ }^{6}$ F. S. Rusin and G. D. Bogomolov, Proc. IEEE Lett. 57, 720 (1969).

${ }^{7}$ E. M. Marshall, P. M. Phillips, and J. E. Walsh, IEEE Trans. Plasma Sci. PS-16, 199 (1988).
${ }^{8}$ R. F. Harrington, Time-Harmonic Electromagnetic Fields (McGrawHill, New York, 1961).

${ }^{9}$ J. H. Booske, B. D. McVey, and T. M. Antonsen, Jr., J. Appl. Phys. 73, 4140 (1993).

${ }^{10}$ R. J. Briggs, Electron-Stream Interaction with Plasmas (MIT Press, Cambridge, MA, 1964).

"M. M. Ali, K. Ogura, K. Minami, T. Watanabe, W. W. Destler, and V. L. Granatstein, Phys. Fluids B 4, 1023 (1992).

${ }^{12}$ The GRAMP code is written to solve for linear growth rates and to generate contour plots of $-D(\omega, k)$-for the beam-grating interactions. The Briggs' criteria are utilized to analyze the contour plots to determine absolute and convective wave growth for the system.

${ }^{13}$ R. E. Collin, Field Theory of Guided Waves (McGraw-Hill, New York, 1960), pp. 225-229.

${ }^{14}$ R. E. Collin, Foundations of Microwave Engineering (McGraw-Hill, New York, 1966), pp. 381-383.

${ }^{15}$ B. D. McVey, M. A. Basten, J. H. Booske, J. Joe, and J. E. Scharer, "Analysis of rectangular waveguide gratings for amplifier applications," to appear in IEEE Trans. Microwave Theory Tech. (1994).

${ }^{16}$ D. A. Watkins, Topics in Electromagnetic Theory (Wiley, New York, 1958), p. 21.

17 J. R. Pierce, Traveling-Wave Tubes (Van Nostrand, New York, 1950).

${ }^{18}$ G. D. Simms and I. M. Stephenson, Microwave Tubes and Semiconductor Devices (Blackie, Glasgow, 1963), p. 67. 